\title{
Drosophila mutants show NMD pathway activity is reduced, but not eliminated, in the absence of Smg6
}

\author{
KIMBERLY A. FRIZZELL, SHAWN G. RYNEARSON, and MARK M. METZSTEIN ${ }^{\mathbf{1}}$ \\ Department of Human Genetics, University of Utah, Salt Lake City, Utah 84112, USA
}

\begin{abstract}
The nonsense-mediated mRNA decay (NMD) pathway is best known for targeting mutant mRNAs containing premature termination codons for rapid degradation, but it is also required for regulation of many endogenous transcripts. Components of the NMD pathway were originally identified by forward genetic screens in yeast and Caenorhabditis elegans. In other organisms, the NMD pathway has been investigated by studying the homologs of these genes. We present here the first unbiased genetic screen in Drosophila designed specifically to identify genes involved in NMD. By using a highly efficient genetic mosaic approach, we have screened $\sim 40 \%$ of the Drosophila genome and isolated more than 40 alleles of genes required for NMD. We focus on alleles we have obtained in two known NMD components: Upf2 and Smg6. Our analysis of multiple alleles of the core NMD component Upf2 reveals that the Upf2 requirement in NMD may be separate from its requirement for viability, indicating additional critical cellular roles for this protein. Our alleles of Smg6 are the first point mutations obtained in Drosophila, and we find that Smg6 has both endonucleolytic and nonendonucleolytic roles in NMD. Thus, our genetic screens have revealed that Drosophila NMD factors play distinct roles in target regulation, similar to what is found in mammals, but distinct from the relatively similar requirements for NMD genes observed in $C$. elegans and yeast.
\end{abstract}

Keywords: Drosophila; NMD; Smg6; Upf2; nonsense-mediated decay

\section{INTRODUCTION}

Nonsense-mediated mRNA decay (NMD) is a cellular pathway that selectively targets mRNAs containing premature termination codons (PTCs) for rapid degradation (Chang et al. 2007) and is thought to function to prevent the translation and build-up of potentially harmful truncated protein products (Cali and Anderson 1998). Trans-acting factors involved in NMD were originally identified in suppressor screens performed in yeast (Leeds et al. 1991, 1992) and Caenorhabditis elegans (Hodgkin et al. 1989; Cali et al. 1999). These screens took advantage of PTC-containing alleles of genes whose truncated protein products, rather than being toxic, possess residual function. Since NMD targets these transcripts, the truncated proteins cannot be made, effectively resulting in a complete loss-of-function phenotype. Loss of function of genes required for NMD results in stabilization of such PTC-containing transcripts, allowing for production of the truncated protein products and

\footnotetext{
${ }^{1}$ Corresponding author

E-mail markm@genetics.utah.edu

Article published online ahead of print. Article and publication date are at http://www.rnajournal.org/cgi/doi/10.1261/rna.032821.112.
}

amelioration of the mutant phenotype. These screens identified three genes required for NMD in yeast-UPF1, UPF2 (also called NMD2), and UPF3 (Upf, up-frameshift suppressor) (Leeds et al. 1991, 1992)-and seven in $C$. elegans_-smg-1 through smg-7 (smg, suppressor with morphogenetic effect on genitalia) (Hodgkin et al. 1989; Cali et al. 1999). Cloning of these genes has revealed that UPF1, UPF2, and UPF3 are orthologous to smg-2, smg-3, and $s m g-4$, respectively, thus defining an evolutionarily conserved core machinery for NMD (Cui et al. 1995; He and Jacobson 1995; Lee and Culbertson 1995; Cali et al. 1999; Page et al. 1999; Aronoff et al. 2001; Serin et al. 2001; Chiu et al. 2003; Grimson et al. 2004). All eukaryotes analyzed contain orthologs of these core genes, and, in all cases examined, they are known to be required for NMD (Kerényi et al. 2008). The four other genes identified in C. elegans (smg-1, smg-5, smg-6, smg-7) are generally conserved only in metazoa, although there is evidence that a homolog of $5 m g 7$ is also required for NMD in plants (Luke et al. 2007). Smg5 and Smg7 are structurally similar (Fukuhara et al. 2005), although not redundant for their function in NMD, and some organisms, including Drosophila, appear only to possess Smg5 (Gatfield et al. 2003). 
The design of the suppressor screens used to identify NMD genes demanded that compromising NMD function would not in itself result in lethality. Indeed, this is the case in yeast and C. elegans: Complete absence of core NMD components and NMD activity is compatible with viability (Leeds et al. 1991, 1992; Cui et al. 1995; He and Jacobson 1995; Lee and Culbertson 1995; Cali et al. 1999; Page et al. 1999; Aronoff et al. 2001; Grimson et al. 2004). However, for most eukaryotes, disruption of NMD gene function is lethal (Hwang and Maquat 2011), and this essential role has limited the ability to identify NMD genes as suppressors of PTC-containing alleles in these organisms.

Since the initial screens, the only unbiased forward genetic screen that has been performed to identify genes required for NMD has been an RNAi-based approach in $C$. elegans. This screen did not rely on suppression, but instead used an NMD-sensitive reporter whose expression increased upon disruption of NMD activity (Longman et al. 2007). This screen identified two new NMD genes, smgl-1 and smgl-2 (smg lethal), whose knockdown leads to defective NMD and also organismal lethality. Knockdown of smgl-1 and smgl-2 in human cells (Longman et al. 2007) and zebrafish (Anastasaki et al. 2011) also leads to impairment of NMD. However, no mutations in smgl-1 or smgl-2 have been reported in any organism.

The primary mechanism that distinguishes premature from normal termination codons seems to involve detection of $3^{\prime}$-UTR length (Amrani et al. 2004; Longman et al. 2007; Eberle et al. 2008). Such a mechanism also plays a role in mammalian cells and plant cells, but there is also an important contribution from interactions between NMD components and splicing-deposited exon junction complexes (Lejeune and Maquat 2005; Kertész et al. 2006; Eberle et al. 2008). Recognition of a PTC is thought to result in the recruitment of the core NMD protein Upf1 (SMG-2 in C. elegans), an ATP-dependent RNA-helicase, to the targeted mRNA, triggering degradation (Kashima et al. 2006). In both yeast and mammalian cells, degradation is known to occur through deadenylation-independent decapping, dependent on the recruitment of the decapping proteins DCP1 and DCP2 by Upf1 (Lejeune et al. 2003; Swisher and Parker 2011). This is followed by degradation by $5^{\prime}-3^{\prime}$ exonucleases, such as XRN1 (He et al. 2003). However, there is now considerable evidence that in metazoan cells, degradation can also occur through endonucleolytic cleavage in the vicinity of the PTC (Gatfield and Izaurralde 2004; Huntzinger et al. 2008; Eberle et al. 2009). The endonuclease responsible for this cleavage is likely to be the NMD component Smg6. Smg6 contains a C-terminally located PIN-endonuclease domain that is required for cleavage of target mRNAs (Glavan et al. 2006; Huntzinger et al. 2008; Eberle et al. 2009). Smg6 is thought to be recruited to target mRNAs by virtue of its 14-3-3-like domain, which can bind phosphorylated Upf1 (Fukuhara et al. 2005). In mammalian cells, Smg6 is also recruited to target mRNAs through direct recruitment to the EJC (Kashima et al. 2010). Smg6 function has primarily been analyzed in cell culture, and while some genetic analysis has been performed in C. elegans (Hodgkin et al. 1989; Page et al. 1999), a role for SMG-6 endonuclease function has not yet been analyzed in this organism.

Roles of Drosophila NMD factors have been well established in cell culture experiments (Gatfield et al. 2003; Rehwinkel et al. 2005; Huntzinger et al. 2008). These experiments have shown that all the Drosophila homologs of known NMD genes are required for NMD and that loss of any of these genes leads to similar defects in NMD. Less is known about how NMD genes function in intact animals. Previous genetic analysis has identified alleles of Upf1-3 and Smg1 (Chen et al. 2005; Metzstein and Krasnow 2006; Avery et al. 2011). All of these have been shown to have function in NMD, although, interestingly, some of these roles differ. For instance, while $U p f 1$ and $U p f 2$ seem to be absolutely required for NMD and organismal viability, loss of Smg1 or $U p f 3$ leads to viable animals with a reduction, but not a complete loss, of NMD pathway activity. Why intact animals and cell culture show these differences is not understood, but probably indicate additional layers of pathway regulation.

Here, we present a novel forward genetic screen in Drosophila that uses an NMD-sensitive reporter combined with a genetic mosaic approach. This has allowed us to isolate more than 30 mutations in the known NMD genes Upf1, Upf2, Smg1, and Smg6. Our alleles of Upf2 have revealed that differential interactions of Upf2 with other core NMD components are correlated with whether these alleles are required for viability. The Smg6 mutations are the first obtained in Drosophila, and we have used genetic and molecular criteria to show that several of these are complete loss of function. We find that mutants carrying these null alleles still show considerable NMD pathway function, thus revealing that Smg6 is not absolutely required for NMD in vivo.

\section{RESULTS AND DISCUSSION}

\section{Tissue-directed mosaic screen for mutations affecting NMD}

To identify novel genes required for NMD as well as alleles of known NMD genes, we performed a large-scale forward genetic screen in Drosophila. The screen uses the simian virus 40 small $\mathrm{t}$ antigen intron and polyadenylation signal (SV40 3' UTR), which is targeted for degradation by the NMD pathway in Drosophila (Metzstein and Krasnow 2006). Transgenic reporter constructs that use the SV40 3' UTR as their termination signal result in a low level of expression in wild-type backgrounds; however, the signal increases dramatically when genes involved in NMD are inactivated (Fig. 1A,B). Fluorescent protein reporters with 

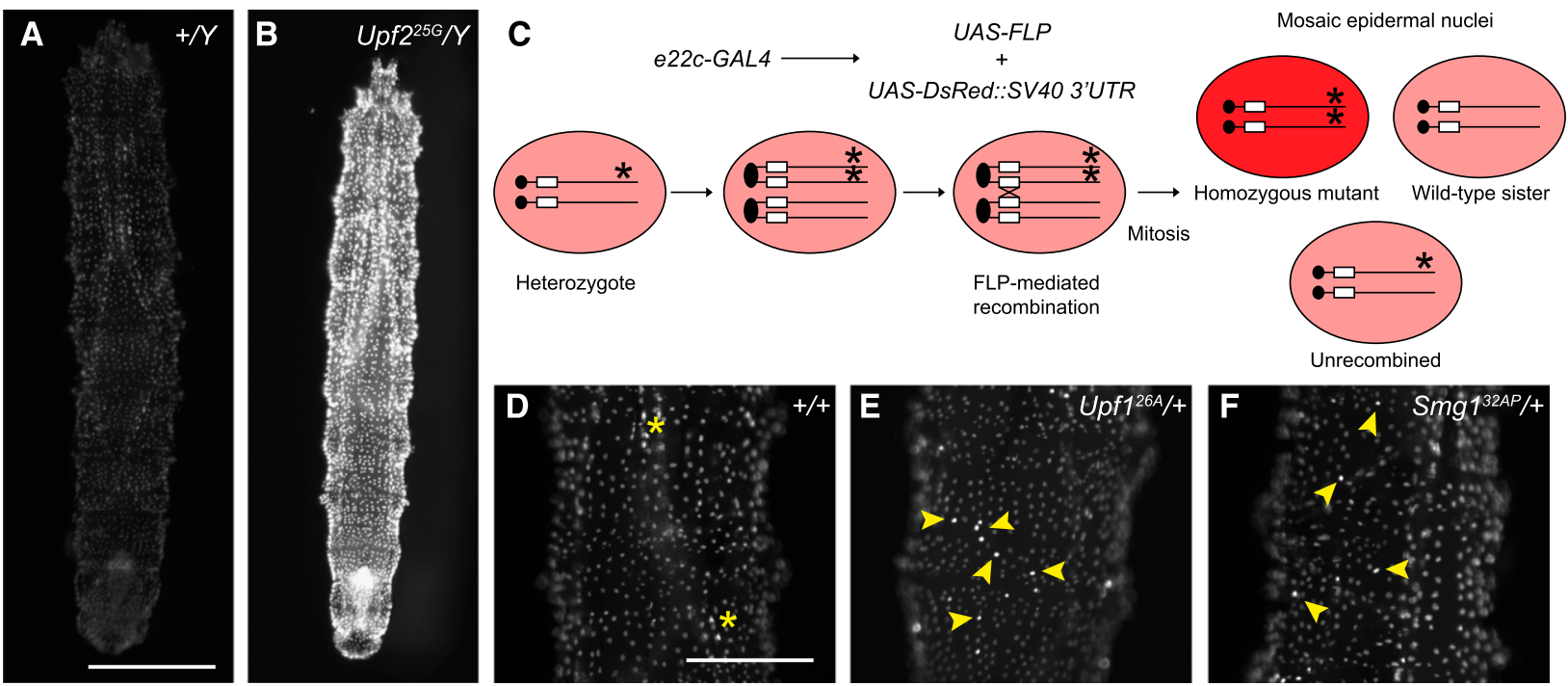

FIGURE 1. Mosaic approach used to detect animals with mutations in genes affecting NMD. (A) y w FRT ${ }^{19 A} / Y$; e22c-GAL4 UAS-nlsDsRed2::SV40 $3^{\prime} U T R /+$ L3 (nonmosaic) larva shows uniform, low-level DsRed expression in epidermal nuclei. (B) y $w$ Upf2 $2^{25 G}$ FRT $T^{19 A} / Y$; e22c-GAL4, UASnlsDsRed2::SV40 3'UTR/+ (nonmosaic) L3 larva, taken at the same exposure as A, shows uniform, high-level DsRed expression in epidermal nuclei, due to stabilization of the reporter mRNA. (C) UAS-FLP expression in the early embryonic ectoderm driven by e22c-GAL4 catalyzes recombination in heterozygous mitotic cells, resulting in homozygous mutant and wild-type sister cells. Cells that have not undergone recombination remain heterozygous. Expression of an NMD-sensitive reporter UAS-nlsDsRed2::SV40 3'UTR, which encodes a nuclearlocalized red-fluorescent protein, is driven by e22c-GAL4 in the larval epidermis. In heterozygous and the wild-type sister cells, the reporter mRNA is targeted for degradation by NMD and shows a low level of DsRed fluorescence (light red). When a gene required for NMD is mutated, the homozygous mutant cell is recognized by an increase in fluorescence (increased red color). (Circles) Centromeres; (rectangles) FRT sites; $\left(^{*}\right)$ EMS-generated mutations. $(D)$ y $w$ FRT ${ }^{19 A}$; e22c-GAL4, UAS-nlsDsRed2::SV40 $3^{\prime} U T R$, UAS-FLP/+ mosaic L3 larva shows uniform DsRed expression in epidermal nuclei. (Yellow asterisks) Underlying tracheal nuclei that also express DsRed. These are easily

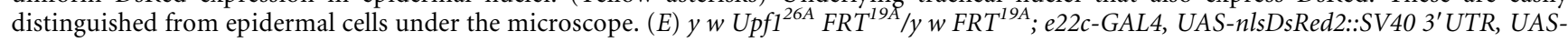
$F L P /+$ L3 larva shows mosaic increased DsRed expression in epidermal nuclei: Low-level DsRed expression is punctuated with brighter, homozygous mutant nuclei (yellow arrowheads). (F) y w Smg1 ${ }^{32 A P} F R T^{19 A} / y w$ FRT ${ }^{19 A}$; e22c-GAL4, UAS-nlsDsRed2::SV40 3'UTR, UAS-FLP/+ L3 larva shows mosaic DsRed expression in epidermal nuclei: Low-level DsRed expression is punctuated with brighter, homozygous mutant nuclei (yellow arrowheads). Scale bars, $(A, B) 1 \mathrm{~mm}$; $(D-F) 0.5 \mathrm{~mm}$.

the SV40 3' UTR can thus be used to monitor NMD pathway activity on a single-cell level, and this allowed us to perform a screen for genes involved in NMD using a genetic mosaic approach. Importantly, this mosaic approach allowed us to bypass the organismal lethality associated with strong loss of NMD function. Furthermore, by using a live-cell marker, we could score for NMD defects in $F_{1}$ generation animals, greatly increasing the screen throughput.

Our screen used the e22c-GAL4 driver, which is expressed in the early embryonic ectoderm and later is restricted to ectodermally derived epithelium (Duffy et al. 1998), including the larval epidermis. This driver served two purposes in our screen (Fig. 1C). Expression of FLP recombinase driven by e22c-GAL4 early in development produces animals with mosaicism in ectodermal derivatives. Later in development, e22c-GAL4 drives expression of an NMD-sensitive fluorescent reporter in larval epidermal cells. Importantly, we found that this expression in epidermal cells is uniform (Fig. 1D), so differences in reporter levels caused by mutations in NMD genes could be readily identified.

To test our mosaic strategy, we crossed our mosaic generating reporter line to animals carrying known mutations in the core NMD components Upf1 and Upf2 (Fig. 1E; data not shown). Animals entirely mutant for null mutations in either of these genes show 100\% early larval lethality and have strong defects in NMD (Metzstein and Krasnow 2006; Avery et al. 2011). We found that animals with tissuedirected mosaics of Upf1 or Upf2 were viable to the L3 larval stage, and we could easily detect increased expression in homozygous epidermal cells, confirming the feasibility of our approach for identifying organismal lethal mutations affecting NMD. We also found that mutants of the NMD auxiliary factor $S m g 1$, which is viable and has weak defects in NMD (Chen et al. 2005; Metzstein and Krasnow 2006; Avery et al. 2011), produced an obvious mosaic phenotype (Fig. 1F). Thus, our strategy should allow us to isolate alleles of both essential and nonessential NMD genes.

We screened for mutations affecting NMD on the $\mathrm{X}$ chromosome and the right arm of the third chromosome using chemical mutagenesis and our mosaic approach (Fig. 2). While we identified our new mutants in mosaic animals, for characterization of their roles in the NMD pathway, we studied the alleles in nonmosaic, homozygous animals. In this way, we were able to determine the degree to which the new mutation is required for NMD. We were also able to assess whether the new mutation was lethal or viable in 


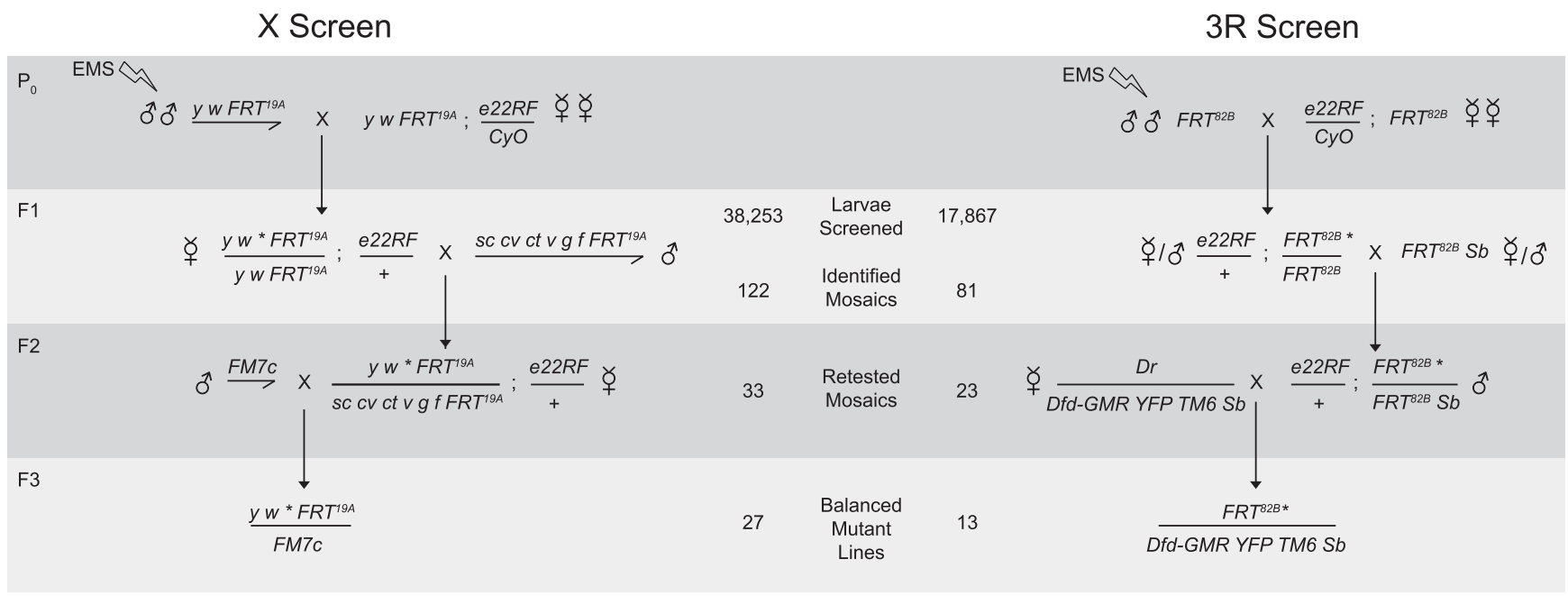

FIGURE 2. Screens to identify genes required for NMD on $X$ and $3 R$. In the $P_{0}$, males carrying the appropriate FRT are mutagenized by treatment with EMS and mated en masse to reporter-containing females. In the $\mathrm{F}_{1}$ generation, candidate NMD-mutant $\mathrm{L} 3$ larvae are identified by mosaic epidermal reporter expression, collected, and individually mated to flies containing marked FRTs. $\mathrm{F}_{2}$ larvae are scored for transmission of the mosaic-expression phenotype, collected, and crossed to balancer-containing animals to establish stocks. (Middle column) Numbers of animals identified in each generation. (e22RF) Genotype e22c-GAL4, UAS-nlsDsRed2::SV40 3'UTR, UAS-FLP.

homozygous animals, giving insight into the requirement of the NMD pathway into organismal survival.

\section{X-chromosome screen has isolated numerous alleles of known NMD genes}

We identified $27 \mathrm{X}$-chromosome mutants, including both lethal and viable alleles (Table 1). Complementation tests revealed that all of these are alleles of the known NMD genes $U p f 1, U p f 2$, and $S m g 1$, in which mutations had been previously isolated (Chen et al. 2005; Metzstein and Krasnow 2006). The most interesting phenotypic diversity we found was in our alleles of Upf2. When tested in homozygous animals, we found that six of these alleles were lethal and six were viable. This phenotypic difference provided us an opportunity to study the effects of Upf2 mutations in vivo. Upf2 is thought to function primarily as a bridge between the other two core components of the NMD complex, Upf1 and Upf3. Binding of Upf2 to Upf3 has been mapped to a conserved MIF4G domain (Fig. 3A), while binding of Upf2 to Upf1 occurs primarily through a C-terminally localized domain, with a contribution from a domain close to the $\mathrm{N}$ terminus (He et al. 1997; Kadlec et al. 2004; Clerici et al. 2009). Upf2 also contains two other MIF4G domains of unknown function (Fig. 3A). To identify which domains of the Upf2 protein are necessary for viability, we sequenced the coding region of $U p f 2$ in eight of our alleles (four lethal and four viable) (Fig. 3A). Our analysis of these $U p f 2$ alleles revealed an interesting pattern: Lethal mutations are predicted to disrupt the second MIF4G domain but leave the other protein domains intact. Two previously characterized lethal alleles of Upf2 are also predicted to disrupt the second MIF4G domain (Metzstein and Krasnow 2006); although these are also protein truncations, this disruption is not very specific. We did recover mutations that are predicted to disrupt the Upf2 interaction with Upf1 and that were not lethal. When we assayed NMD pathway activity in these viable mutants, we found that there was always residual pathway activity (Fig. 3B). These results suggest that pathway activity correlates with viability. This agrees with previous results showing that complete loss of the Upf1/Upf2 interaction is not compatible with viability (Avery et al. 2011). However, we cannot currently rule out that the second MIF4G domain has a cellular function independent of its role in NMD and disruption of this function also results in organismal

TABLE 1. 27 alleles recovered on the $X$ chromosome

\begin{tabular}{|c|c|c|c|c|c|}
\hline \multicolumn{2}{|c|}{ Upf1 } & \multicolumn{2}{|c|}{ Upf2 } & \multicolumn{2}{|c|}{ Smg1 } \\
\hline Allele & Viability & Allele & Viability & Allele & Viability \\
\hline 42 & Lethal & $14-01-3 M$ & Lethal & $7-24-3$ & Viable \\
\hline$A 92 A$ & Lethal & $3-01-3 R$ & Lethal & $8-9 A$ & Viable \\
\hline $8-7 H$ & Lethal & $7-5 A$ & Lethal & $7-10-2$ & Viable \\
\hline \multirow[t]{9}{*}{$14 D$} & Lethal & $C 41$ & Lethal & $11-29-3-1$ & Viable \\
\hline & & C51 & Lethal & $C 9$ & Viable \\
\hline & & $C 2$ & Lethal & C73 & Viable \\
\hline & & $2-8 A$ & Viable & $8 D 2$ & Viable \\
\hline & & $4-5 F$ & Viable & A6 & Viable \\
\hline & & $C 72$ & Viable & A91 & Viable \\
\hline & & $3 D$ & Viable & $6 B$ & Viable \\
\hline & & C52 & Viable & $11-2-10 B$ & Viable \\
\hline & & $13 D$ & Viable & & \\
\hline
\end{tabular}

X-chromosome screen mutants. All alleles of Upf1 are lethal, and all alleles of Smg1 are viable, while alleles of Upf2 can be either lethal or viable. 
A

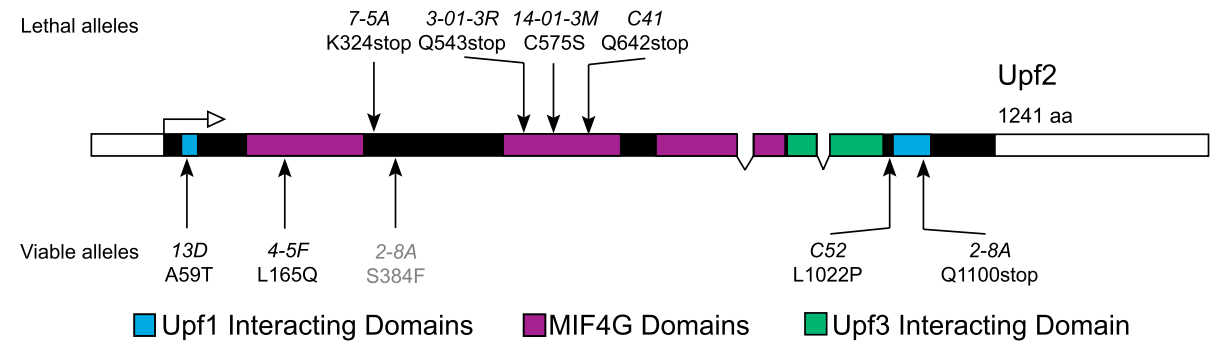

B

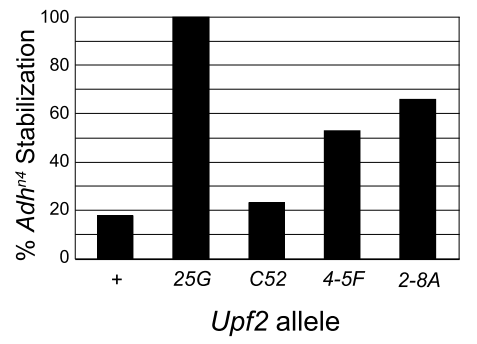

FIGURE 3. Characterization of $U p f 2$ alleles. (A) Mutations in Upf2. Alleles shown above the gene model are hemizygous lethal; those below the gene are hemizygous viable. 2-8A contains two mutations: a late glutamine to stop (black), as well as a serine-to-phenylalanine missense mutation (gray). This latter change is in a nonconserved domain of Upf2, and we suspect it does not contribute to the Upf2 defect. (Filled boxes) Coding sequences; (open boxes) 5' and 3' UTRs; (blue) bipartite Upf1 binding domain; (green) Upf3 binding domain; (purple) MIF4G domains (the third one of which overlaps the Upf3 binding domain); (open arrow) direction of transcription. (B) Effect of viable Upf2 mutations on levels of the PTC-containing transcript $A d h^{n 4}$ (Q83Stop). Values are the proportion of $A d h^{n 4}$ transcript relative to $A d h^{+}$transcript normalized to $U p f 2^{25 G}$, in which $A d h^{n 4}$ is completely stabilized.

lethality. Finally, we noted that three of the four viable Upf2 alleles were male sterile (data not shown), suggesting a role for Upf2 in the male germline (Table 2). Whether this role is directly related to its role in NMD has not yet been determined.

We obtained four alleles of Upf1 but did not find that any of them are compatible with viability (Table 1), suggesting that even subtle perturbations in Upf1 structure are disruptive to protein function. In addition to $U p f 1$ and $U p f 2$, we isolated 11 alleles of $S m g 1$. It has previously been observed that complete loss of Smg1 leads to no effect on viability and only a slight decrease in NMD function (Chen et al. 2005; Metzstein and Krasnow 2006). Indeed, we found that all 11 alleles were viable (Table 1).

Based on the large number of Upf1, Upf2, and Smg1 alleles, we believe that we have saturated the $\mathrm{X}$ chromosome for genes required for NMD and that can be mutated by EMS, the chemical mutagen we used. Notably, we did not isolate any alleles of CG32533, the Drosophila homolog of the C. elegans gene smgl-2 (Longman et al. 2007), which is located on the $\mathrm{X}$ chromosome. In addition to not identifying mutations in CG32533 in our screen, we also tested its function using an RNAi approach (A Chapin and MM Metzstein, unpubl.) and did not detect an NMD defect. Combined, these data suggest that smgl-2 does not play a significant role in NMD in Drosophila. However, since EMS mutagenesis and RNAi knockdown do not affect all genes equally, it will be necessary to obtain mutations in smgl-2 by some other means to definitively test the role of this gene in NMD in Drosophila.

\section{Third chromosome screen has isolated first Drosophila alleles of $5 m g 6$}

We screened the right arm of the third chromosome and identified 13 mutants. Similar to the X chromosome, after identifying the mutants with our mosaic approach, we studied the role of the genes affected in wholly mutant animals. By a complementation test with a chromosomal deficiency, we found that six of our 13 lines were likely to be mutations in Smg6 (Supplemental Fig. S1). In all cases, the Smg6 mutation was viable in trans to the deficiency. These six alleles also failed to complement each other (using the assay of reporter expression) (Supplemental Fig. S1). Three of the lines were viable as homozygotes. However, the remaining three lines were homozygous lethal, suggesting that extraneous lethal mutations are present on these chromosomes. The other seven lines complemented the deficiency for both viability and reporter enhancement and will be characterized further in a later study.

To identify the molecular changes in our candidate Smg6 alleles, we sequenced the $S m g 6$ genomic locus in each of the six mutant lines. A single mutation affecting the Smg6 gene was identified in each line (Fig. 4A; Table 3). The identified lesions are spread throughout the length of the Smg6 gene and include premature termination codons, splice mutations, and a single missense change. This missense change,

TABLE 2. Molecular analysis of Upf2 alleles

\begin{tabular}{lllll}
\hline Allele & Viability & Mutation & Residue change & Male fertile \\
\hline 14-01-3M & Lethal & T1723A & C575S & N.A. \\
$3-01-3 R$ & Lethal & G1627A & Q543Stop & N.A. \\
$7-5 A$ & Lethal & A1694T & K324Stop & N.A. \\
C41 & Lethal & G1924A & Q642Stop & N.A. \\
$2-8 A$ & Viable & C1876T & S384F & No \\
& & C3298T & Q1100Stop & \\
$4-5 F$ & Viable & T494A & L165Q & No \\
C52 & Viable & T3065C & L1022P & No \\
$13 D$ & Viable & G175A & A59T & Yes \\
\hline
\end{tabular}

For mutation positions, bases are numbered with the $A$ of the start codon as 1 , and introns are included. 7-5A appears to be identical to the previously isolated Upf2 allele 29AA (Metzstein and Krasnow 2006). 


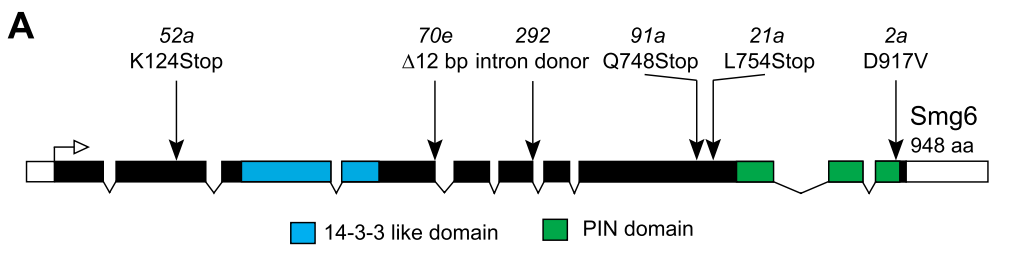

B

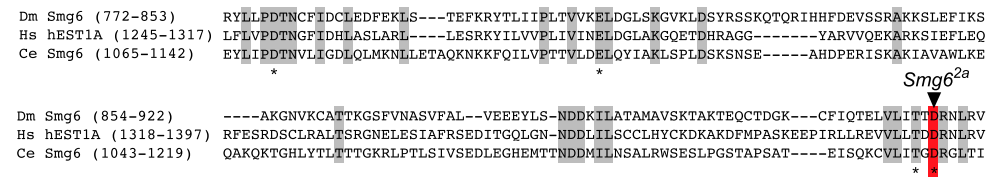

C

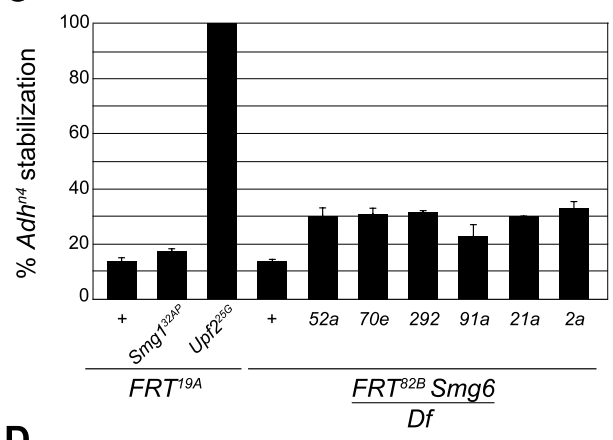

D

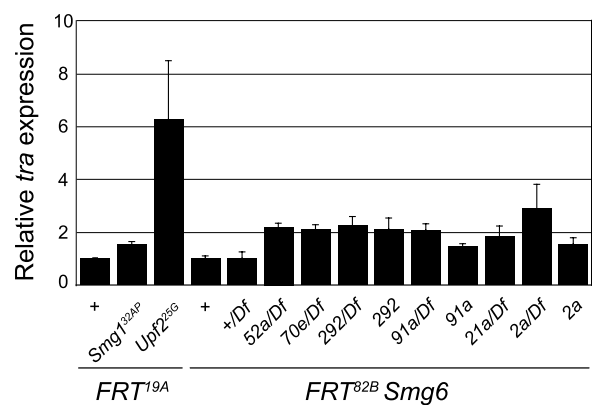

$\mathbf{E}$

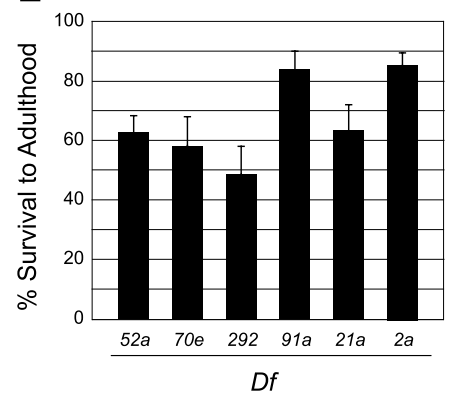

$\mathbf{F}$

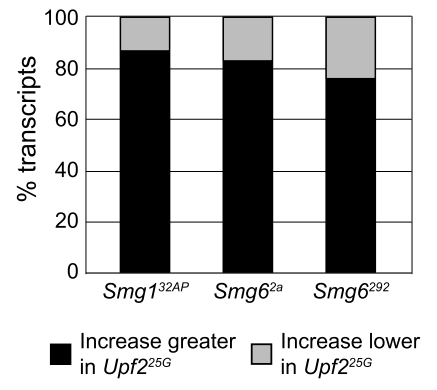

FIGURE 4. Molecular and phenotypic characterization of Smg6 alleles. (A) Mutations in Smg6. (Filled boxes) Coding sequences; (open boxes) 5' and 3' UTRs; (blue) 14-3-3-like protein binding domain; (green) PIN endonucleolytic cleavage domain; (open arrow) direction of transcription. (B) Alignment of Smg6 PIN domains. These highly conserved domains are representative of PIN domains found in Smg6 and other PIN-domaincontaining proteins (Takeshita et al. 2007). (Dm) D. melanogaster; (Hs) Homo sapiens; (Ce) C. elegans. $\left(^{*}\right.$ ) Invariant catalytic residues; (gray shading) residues conserved in all three species; (red shading) aspartic acid residue altered to a valine in $S m g 6^{2 a}$. (C) Effect of $S m g 6$ mutations on levels of the PTC-containing transcript $A d h^{n 4}$ (Q83Stop). Values are the proportion of $A d h^{n 4}$ transcript relative to $A d h^{+}$transcript normalized to $U p f 2^{25 G}$, in which the $A d h^{n 4}$ is completely stabilized. Error bars indicate SD, $n=2$. (D) Effect of Smg6 mutations on levels of the endogenous NMD target tra in males. Levels for Smg6 alleles are normalized to $F R T^{82 B} / D f$ animals; Smg $1^{32 A P} / Y$ and $U p f 2^{25 G} / Y$ are normalized to $F R T^{19 A} / Y$. Error bars indicate SEM, $n=3$. (E) Percentage survival to adulthood of Smg6 mutants. Each of the alleles are in trans to an Smg6 deficiency. The percentage is calculated relative to balancer-containing siblings. Error bars: $95 \%$ confidence interval of the binomial distribution. $(F)$ Microarray analysis of relative magnitude of NMD defects observed in $U p f 2^{25 G}, S m g 1^{32 A P}$, and two Smg6 alleles, Smg6 $6^{2 a}$ and $S m g 6^{292}$. For each mutant genotype, we calculated transcript expression relative to its respective wild-type control. To compare the effect of different NMD genes with transcript regulation, we first extracted targets that increased more than twofold in all four of our NMD-mutant conditions, giving a list of 87 genes (Supplemental Table S1). We then compared the magnitude of the increase observed in Smg1 $1^{32 A P}, S m g 6^{2 a}$, and Smg6 ${ }^{292}$ with that observed in $U p f 2^{25 G}$. (Black bars) Transcripts that increased more in $U p f 2^{25 G}$ relative to transcripts in Smg1 or Smg6; (gray bars) transcripts that increase more in $S m g 1$ or Simg6 alleles relative to Upf2 $2^{25 G}$.

found in the allele $S m g 6^{2 a}$, is of particular interest, because it alters an invariantly conserved aspartate residue found in all PIN domains (Fig. 4B) and is known from structural studies to be part of the endonucleolytic catalytic core (Glavan et al. 2006; Takeshita et al. 2007).

\section{Complete loss of Smg6 still shows NMD-pathway activity in Drosophila}

To characterize the role of Drosophila Smg6 in NMD, we analyzed mRNA levels of the well-characterized NMD 
TABLE 3. Molecular analysis of Smg6 alleles

\begin{tabular}{lll}
\hline Allele & Base change & Residue change \\
\hline $52 a$ & A535T & K124Stop \\
$70 e$ & GAgtgagtatat465 & N.A. \\
292 & g558a & N.A. \\
$91 a$ & C2775T & Q748Stop \\
$21 a$ & T2794A & L754Stop \\
$2 a$ & A3580T & D917V \\
\hline
\end{tabular}

For mutation positions, bases are numbered with the $\mathrm{A}$ of the start codon as 1, and introns are included. $70 \mathrm{e}$ is a 12-bp deletion that removes the last two bases of the fourth exon (uppercase) and $10 \mathrm{bp}$ of the fourth intron (lowercase). 292 is a point mutation that converts the $G$ of the sixth intron splice donor to an A.

target gene $A d h^{n 4}$, which contains a nonsense mutation (Chia et al. 1987; Brogna 1999). $A d h^{n 4}$ has been shown to be a bona fide NMD target in both cell culture and whole animals (Brogna 1999; Gatfield et al. 2003; Chen et al. 2005; Metzstein and Krasnow 2006). Furthermore, Adh is expressed in most, if not all Drosophila tissues (Chintapalli et al. 2007), so by analyzing this gene we can assess the role played by NMD in diverse cell types.

Steady-state mRNA levels depend on mRNA stability and are thus a proxy for decay rates, and in $A d h^{n 4} / A d h^{+}$ animals, $A d h^{n 4}$ mRNA is expressed at $\sim 10 \%$ of the amount of the $A d h^{+}$transcript, due to NMD-dependent destabilization of the mutant transcript (Brogna 1999; Chen et al. 2005; Metzstein and Krasnow 2006). We found that loss of Smg6 function results in stabilization of the $A d h^{n 4}$ transcript to $\sim 30 \%$ of the amount of $A d h^{+}$transcript (Fig. 4C). A similar degree of stabilization was observed in each of the six Smg6 alleles in trans to a deficiency (Fig. 4C). In comparison, as previously reported, we found that $S m g 1$ mutants had only a very slight effect on $A d h^{n 4}$ levels (Chen et al. 2005; Metzstein and Krasnow 2006), whereas Upf2 ${ }^{25 G}$, a strong hypomorphic allele of the core NMD component $U p f 2$ that essentially abolishes NMD, leads to complete stabilization of $A d h^{n 4}$ (Metzstein and Krasnow 2006). Loss of Smg6 leads to considerably higher levels of $A d h^{n 4}$ than loss of Smg1, but significantly, stabilization of $A d h^{n 4}$ in Smg6 is not nearly as strong as that observed in $U p f 2^{25 G}$. This suggests that Smg6 is not absolutely required for NMD in vivo. We found similar results analyzing a PTC-containing allele of the developmental gene eyes absent (eya) (Supplemental Fig. S2A).

To determine if any of our Smg6 alleles are definitively null, we used the classic genetic test of comparing homozygotes to the allele in trans to a deficiency. For this, we used quantitative reverse transcription coupled PCR (qRT-PCR) to measure the levels of the endogenous NMD-target gene transformer (tra) in Smg6 mutant males (Fig. 4D) (this analysis was possible using the alleles $292,91 a$, and $2 a$, since these were not associated with any linked lethal mutation). Like Adh, tra is broadly expressed in Drosophila tissues and has been shown to be a bona fide NMD target in both intact animals (Metzstein and Krasnow 2006; Avery et al. 2011) and S2 cell culture (Rehwinkel et al. 2005).

Similar to what we observed for $A d h^{n 4}$, we found that loss of Smg6 function results in increased expression of tra to a level intermediate between Smg1 and $U p f 2^{25 G}$. Of particular note, we observed that the degree of stabilization of $\operatorname{tra}$ was the same in $S m g 6^{292}$ homozygotes and $S m g 6^{292} /$ $D f$ hemizygotes, defining this as a null allele. Additionally, one of our other alleles, $\operatorname{Smg} 6^{52 a}$, is predicted be a molecular null, because it contains an early stop codon truncating the coding region of Smg6 before any known functional domains. Although we could not examine Smg6 ${ }^{52 a}$ homozygotes due to a linked lethal mutation, we did find that Smg6 $6^{52 a}$ displays a similar degree of tra stabilization in trans to a deficiency, as does $S m g 6^{292}$. These results were confirmed using two other endogenous NMD targets, Smg5 and Oda (Supplemental Fig. S2B; Gatfield and Izaurralde 2004; Rehwinkel et al. 2005; Avery et al. 2011). Thus, by both molecular and genetic criteria, we have isolated the first definitively null alleles of Smg6.

Interestingly, two of the Smg6 alleles that specifically disrupt the PIN domain seem to retain residual activity. Smg6 ${ }^{91 a}$, a nonsense mutation just preceding the PIN domain, and $S m g 6^{2 a}$, the missense mutation in the PIN domain, have lower levels of tra stabilization than null alleles, and this stabilization is increased when these alleles are placed in trans to a deficiency (Fig. 4D; Supplemental Fig. S2B). These results suggest that Smg6 has some activity independent of the PIN domain and thus presumably independent of its role as an endonuclease.

In general, the severity of NMD mutations and organismal viability are correlated in Drosophila. The NMD genes Upf1 and Upf2 are lethal as null alleles in Drosophila. The strong hypomorphic allele of $U p f 2^{25 G}$ is strongly disrupted in NMD function, and mutant animals are only partially viable, while a deletion of Upf3 has moderate effects on NMD and is viable (Avery et al. 2011). Null alleles of Smg1 have mild effects on NMD and are fully viable (Chen et al. 2005; Metzstein and Krasnow 2006). We therefore investigated the requirement for $S m g 6$ for viability by analyzing each of the Smg6 alleles in trans to a deficiency for their survival to adulthood. In each case, loss of Smg6 leads to only a moderate reduction in viability (Fig. 4E), further demonstrating that Smg6 is not absolutely required for NMD in Drosophila.

To determine on a more global level whether loss of Smg6 activity had less effect on the NMD pathway than loss of $U p f 2$, we performed a microarray analysis. RNA was extracted from $U p f 2^{25 G}, S m g 6^{2 a}$, and $S m g 6^{292} \mathrm{mu}$ tant animals and hybridized to microarrays representing the complete Drosophila transcriptome. We then tabulated genes that increased twofold over matched wildtype controls in all of our mutant conditions (Supplemental Table S1). We found that the large majority of 
such genes had increased expression levels in $U p f 2^{25 G}$ mutants higher than the increase observed in Smg6 mutants (Fig. 4F). From this, we conclude that, for the majority of endogenous NMD targets, Smg6 mutants still possess residual NMD-pathway activity. When we tested Smg $1^{32 A P}$ by the same assay, we found that it led to generally lower levels of transcript stabilization compared with Smg6 (Fig. 4F). These results are consistent with our analysis of candidate transcripts, described above.

Another phenotype associated with loss of NMD function in Drosophila is impairment of cell proliferation, both in S2 cell culture as well as in vivo (Rehwinkel et al. 2005; Metzstein and Krasnow 2006; Avery et al. 2011). We examined cell proliferation mosaic analysis in the eye, a technique that permits comparison of homozygous mutant cell growth in the presence or absence of wildtype cells (Stowers and Schwarz 1999). We find that, similar to other NMD mutants (Metzstein and Krasnow 2006; Avery et al. 2011), Smg6 mutant cells are defective in cell proliferation when in competition with wild-type cells, as assayed by examination of mitotic clones within the eye (Supplemental Fig. S4A-D). However, when competing cells are removed, Smg6 mutant cells can essentially proliferate as well as wild-type cells (Supplemental Fig. S4E-H). These data show that while Smg6 is dispensable for viability, it is still required for aspects of cell function in vivo.

\section{Conclusions}

We have isolated point mutations in Drosophila Smg6, which are the first mutant alleles of Smg6 characterized in any organism since the initial identification of this gene in C. elegans (Hodgkin et al. 1989). Since a number of our Smg6 alleles clearly lack all function, this has allowed us to assess the contribution of this gene to the NMD process in vivo. We find that in Drosophila, Smg6 has only a moderate role in targeting PTC-containing transcripts for degradation. In comparison, mutations in core NMD components, such as $U p f 2$, lead to complete stabilization of NMD targets in vivo (Metzstein and Krasnow 2006; Avery et al. 2011). Our results contrast with previous analysis of Smg6 in Drosophila S2 cells using RNAi-mediated knockdown, in which inactivation of Smg6 appeared to result in as strong an effect on NMD targets as inactivation of other components of the NMD pathway (Gatfield et al. 2003; Rehwinkel et al. 2005). One explanation for this contradiction is that it is not possible to assess the exact degree of gene inactivation in RNAi knockdown experiments, making comparisons of the requirements of different genes in a process difficult to assess.

It is interesting to compare our results of $5 m g 6$ mutations in vivo with the study of this gene in other species. In C. elegans, the organism in which Smg6 was first identified, the NMD process seems to be as dependent on Smg6 as any of the other NMD genes (Hodgkin et al. 1989). However, because no description of the molecular nature of C. elegans Smg6 alleles has been published, it is unknown if any of the known alleles are null. Indeed, there is some evidence that null alleles of $C$. elegans smg- 6 are not viable, thus the alleles allowing viable NMD-defective animals may indeed possess residual function (Cali and Anderson 1998). In zebrafish, Smg6 has been analyzed by morpholino knockdown in intact fish and by expression of an Smg6 dominant-negative construct in fish cell lines (Wittkopp et al. 2009). The morpholino knockdown behaves very similarly to knockdown of other NMD components, leading to early lethality with distinct patterning and neural defects, suggesting that Smg6 has similar roles to these other components in vivo. However, because direct measurement of NMD-pathway activity was not performed in the intact Smg6 morphants, it is possible that there is still residual pathway activity, as observed in Drosophila. Measurements of NMD-pathway activity were analyzed in fish cell culture and clearly show a defect in NMD. Whether this defect is equivalent to complete NMD-pathway loss was not analyzed.

Smg6 is an endonuclease that cleaves target mRNAs around the site of the premature termination codon (Huntzinger et al. 2008), and our data support this as an important Smg6 function. All of our alleles of Smg6 are predicted to disrupt the C-terminal endonucleolytic PIN domain, including one that is a point mutation affecting a key catalytic residue. However, since Smg6-null mutants still show considerable NMD-pathway activity, this suggests that in intact Drosophila there exists an Smg6-independent degradation pathway. In yeast, NMD targets are thought to be degraded by decapping followed by $5^{\prime}-3^{\prime}$ exonucleolytic degradation (Muhlrad and Parker 1994; He et al. 2003). Inactivation of this pathway was shown not to have an effect on NMD-mediated degradation in S2 cells (Gatfield et al. 2003), but one possibility is that this mechanism is only used in the absence of Smg6-mediated cleavage. Indeed, mammalian cells are known to use multiple decay pathways (Chen and Shyu 2003; Lejeune et al. 2003; Huang et al. 2011). Drosophila genetics may provide a powerful system for identifying such alternative degradation pathways, because mutations in such genes would be predicted to act as enhancers of the Smg6 phenotype.

Like Smg6, Smg1 is not absolutely required for NMD in Drosophila (Chen et al. 2005; Metzstein and Krasnow 2006). Smg1 is a PIKK-family kinase that phosphorylates the core NMD component Upf1 to provide a binding platform for Smg6 (Kashima et al. 2006; Okada-Katsuhata et al. 2012). If such binding were absolutely necessary for Smg6 activity, we would expect that Smg1 mutations would have at least as strong an NMD defect as Smg6 mutations. However, we find just the opposite: Loss of Smg6 activity leads to a significantly stronger NMD defect than loss of Smg1, suggesting that Smg6 has activity independent of 
Smg1-mediated phosphorylation of Upf1. In mammalian cells, Smg6 can interact with the NMD complex independent of binding to phosphorylated Upf1 through interactions between two N-terminal exon junction complex binding domains (Kashima et al. 2010). Drosophila is thought to lack these domains, but it remains possible that Upf1phosphorylation-independent interactions can still occur. Alternatively, the Drosophila genome may contain an alternative kinase redundant with Smg1 that can phosphorylate Upf1. This could explain why Smg1 has a relatively weak affect on NMD in intact Drosophila and provide an alternative mechanism of Smg6 entry into the NMD complex. It should be possible to identify such a kinase by testing other kinases in the Drosophila genome for synergistic effects with Smg1 mutants on the NMD pathway.

Analysis of NMD in mammalian cells has suggested that NMD is not a linear pathway, but potentially different branches act in parallel to robustly regulate the abundance of target mRNAs. For instance, there exist Upf3-dependent and Upf3-independent NMD pathways targeting certain nonsense transcripts in mammalian cells (Chan et al. 2007; Yepiskoposyan et al. 2011). Our genetic screen and molecular analysis presented here has also revealed that multiple mechanisms function to regulate NMD in Drosophila. Finally, the efficient screening method for NMD genes presented here should be applicable to the entire Drosophila genome, providing the opportunity to identify all of the components contributing to these parallel mechanisms.

\section{MATERIALS AND METHODS}

\section{Fly stocks}

Drosophila melanogaster lines were raised at $25^{\circ} \mathrm{C}$ on cornmeal/ dextrose medium using standard conditions. Control chromosomes used for the screens were $y w F R T^{19 A}$ and $F R T^{82 B}$ (Xu and Rubin 1993); the e22c-GAL4 and UAS-FLP stocks (Duffy et al. 1998) were used in all screen stocks to induce epidermal mosaics using the GAL4/UAS system (Brand and Perrimon 1993) and FLP/FRT systems (Xu and Rubin 1993). The nlsDsRed2::SV40 3'UTR reporter transgene was provided by M. Galko (M.D. Anderson Cancer Center). e22c-GAL4, UAS-nlsDsRed2::SV40 3'UTR, UAS-FLP was built using standard recombination methods (MM Metzstein, unpubl.). NMD alleles Upf2 ${ }^{25 G}$, $S m g 1^{32 A P}$, and $U p f 1^{26 A}$ have been described previously (Metzstein and Krasnow 2006). The breathless promoter btl-Gal4 was used in combination with UAS-GFP as the reporter in the X-chromosome complementation tests (Shiga et al. 1996). For the balancer chromosomes, we used $w^{*} ; r y^{506} D^{1} / T M 6 B, P\{D f d$-EYFP $\} S b^{1}$ $c a^{1}$ (Le et al. 2006) and FM7i, P\{ActGFP $\} J M R 3$ (Reichhart and Ferrandon 1998). Complementation tests and deficiency experiments for Smg6 were performed using $D f(3 R) E D 6220 / T M 6 c$ $T b, S b$ (Ryder et al. 2007). Eye clones were generated using $w$; ey-FLP; FRT $T^{82 B} P\{w+r y+\} 90 E$ and $y w ; e y-G A L 4, U A S-F L P$; $F R T^{82 B}$ GMR-hid/TM6 (Stowers and Schwarz 1999). For NMD tests, we used the PTC-containing alleles $A d h^{n 4}$ (Chia et al. 1987) and eya ${ }^{c l i-I I D}$ (Bui et al. 2000).

\section{Mutagenesis and screen scheme}

For mutagenesis, isogenized males were aged 1-3 d, starved overnight, and fed $25 \mathrm{mM}$ ethane methylsulfonate in $1 \%$ sucrose for $24 \mathrm{~h}$ (Newsome et al. 2000). For the X-chromosome screen, y $w F R T^{19 A} / Y$ mutagenized males were mated en masse to $y w$ FRT ${ }^{19 A}$; e22c-GAL4, UAS-nlsDsRed2::SV40 3'UTR, UAS-FLP/ $\mathrm{CyO}$ virgins. $\mathrm{F}_{1}$ wandering third-instar larvae were collected in glycerol and analyzed using a Leica MZ16 fluorescence stereomicroscope. NMD mutants were identified by mosaic enhancement of the DsRed reporter in the larval epidermis. Candidate mutant $F_{1}$ larvae were collected, rinsed in $1 \times \mathrm{PBS}$, placed in individual vials, and allowed to complete development. Surviving $\mathrm{F}_{1}$ 's were mated to $s c c v c t v g f F R T^{19 A} / Y$, and $\mathrm{F}_{2}$ progeny were retested for mosaic enhancement of DsRed expression; mosaic animals were collected and mated to $F M 7 c / Y$ males to establish balanced mutant lines. We screened about 38,000 DsRed-expressing $\mathrm{F}_{1}$ animals. Of these, we expected $50 \%$ to be females, which could exhibit mosaicism (the others are hemizygous males and thus would not be expected to undergo FLPmediated recombination). We identified 122 mosaic larvae from which we recovered 27 balanced mutant lines, representing a recovery rate of $\sim 22 \%$.

For the third chromosome, mutagenized $w / Y$; FRT ${ }^{82 B}$ males were mated en masse to e22c-GAL4, UAS-nlsDsRed2::SV40 3'UTR, $U A S-F L P / C y O ; F R T^{82 B}$ virgins, and $\mathrm{F}_{1}$ animals were scored and collected as described above. Candidate mutants were retested by mating to $y w ; F R T^{82 B} \mathrm{Sb}^{1} / \mathrm{TM} 6 \mathrm{~B} r \mathrm{~Tb} b^{+}$animals of the opposite sex, and male progeny that retested were collected and mated to $w$; $\mathrm{Kr} / \mathrm{CyO}$; D/ TM6c Tb, Sb or to $w^{*} ; r^{506} \mathrm{Dr}^{1} / \mathrm{TM} 6 \mathrm{~B}, \mathrm{P}\{\mathrm{Dfd}-\mathrm{EYFP}\}$, $\mathrm{Sb}^{1} \mathrm{ca}^{1}$ to establish mutant lines. We scored about $17,000 \mathrm{~F}_{1}$ 's (both males and females would be expected to be mosaics), and we identified 81 mosaic larvae. Of these we were able to establish 13 balanced mutant lines, representing a recovery rate of $\sim 16 \%$.

\section{Complementation tests}

$x$

There are three known NMD genes on the $\mathrm{X}$ chromosome with previously isolated alleles (Metzstein and Krasnow 2006). Null alleles of $U p f 1$ and $U p f 2$ are lethal, whereas null alleles of $S m g 1$ are viable (Chen et al. 2005; Metzstein and Krasnow 2006). Complementation tests were performed using these alleles. For Upf1 complementation tests, $y$ w FRT ${ }^{19 A}{ }^{\star} / F M 7 c$ (where * indicates EMS-induced mutation) virgins were mated to $y w$ Upf1 ${ }^{13 D}$ $F R T^{19 A} / D p(1 ; Y) B S C 1\left[U p f 1^{+}\right]$males, and $\mathrm{F}_{1}$ progeny were scored for the absence of $\mathrm{B}^{+}$females, which indicates failure to complement $U p f 1$ for lethality. In cases in which complementation for lethality occurred, the mutant lines were then crossed to $y w$ Upf1 ${ }^{13 D} F R T^{19 A} / D p(1 ; Y) B S C 1\left[U p f 1^{+}\right] ;$Btl-Gal4, UAS-GFP::SV40 $3^{\prime} U T R /+$ males, and $\mathrm{F}_{1}$ female third-instar larvae were scored for enhancement of GFP. Failure to complement this phenotype would have indicated that the candidate gene is a viable Upf1 hypomorph. However, we did not find any alleles of this class.

For $U p f 2$ complementation tests, $y w F R T^{\star} / F M 7 c$ virgins were crossed to $y w U p f 2^{14 J}$ v $g f F R T^{19 A} / Y ; P\left\{w^{+}, U p f 2^{+}\right\} /+$males (Metzstein and Krasnow 2006), and $F_{1}$ animals were scored for the absence of $w B^{+}$females, which indicates failure to complement $U p f 2$ for lethality. In cases in which complementation occurred, 
the mutant lines were crossed to $y w U p f 2^{14 J} v g f F R T^{19 A} / Y ; P\left\{w^{+}\right.$, $\left.U p f 2^{+}\right\} / B t l-G a l 4, U A S-G F P:: S V 40 \quad 3^{\prime} U T R$ males, and $\mathrm{F}_{1}$ female third-instar larvae were scored for GFP enhancement. Failure to complement indicates that the Upf2 allele is a viable hypomorph. For Smg1 complementation tests, females from mutant lines were crossed to $y w S m g 1^{32 A P} F R T^{19 A} / Y$; Btl-Gal4, UAS-GFP::SV40 $3^{\prime} U T R /+$ male larvae and were scored for GFP enhancement, indicating failure to complement Smg1.

\section{$3 R$}

Complementation tests were performed by crossing e22c-GAL4, UAS-nlsDsRed2::SV40 3'UTR, UAS-FLP/CyO; FRT 3 $^{8 B_{*}} / \mathrm{TM} 6 \mathrm{c}$ Tb, $S b$ females to $D f(3 R) E D 6220 / T M 6 c T b, S b$ animals, and thirdinstar larvae were scored for DsRed enhancement. Lines that failed to complement Smg6 were intercrossed to determine if the Smg6 mutations were lethal or had additional linked lethals.

\section{RNA isolation and quantitative RT-PCR}

Total RNA was isolated from five to 10 flash-frozen samples of adult males using TRIzol reagent (Invitrogen) and phase-lock tubes (5-prime), and the RNeasy mini kit (QIAGEN). Genomic contamination was reduced by on-column DNase treatment (Ambion). RNA concentration was determined using a spectrophotometer and normalized for reverse transcription using the MuMulv reverse transcriptase Retroscript Kit (Ambion). Quantitative RTPCR was performed on a Bio-Rad iCycler thermocycler using SYBR Green Supermix (Bio-Rad). All experimental reactions were performed in triplicate with a minimum of three biological replicates. For quantitation, samples were normalized to RpL32.

\section{Analysis of $\mathrm{Adh}^{\mathrm{n} 4}$}

$A d h^{n 4} / C y O$ animals were mated to $D f(3 R) E D 6220 / T M 6 B, P\{D f d-$ EYFP $S b^{1} c a^{1}$. Males in the $\mathrm{F}_{1}$ generation $A d h^{n 4} / A d h^{+}$; $D f(3 R) E D 6220 /+$ were collected and mated to virgin $F R T^{82 B}$ / TM6B, $P\{D f d-E Y F P\} S b^{1} c a^{1}$ or $F R T^{82 B} S m g 6^{*} / T M 6 B, P\{D f d-$ EYFP $S b^{1} c a^{1}$ flies. Animals of genotype $A d h^{n 4} / A d h^{+} ; F R T^{82 B}$ Smg6 $6^{*} / D f(3 R) E D 6220$ and $A d h^{n 4} / A d h^{+} ; F R T^{82 B} / D f(3 R) E D 6220$ were collected by genotyping for the presence of the $A d h^{n 4}$. Animals of genotypes $S m g 1^{32 A P} / Y ; A d h^{n 4} / A d h^{+}$and $U p f 2^{25 G} / Y$; $A d h^{n 4} / A d h^{+}$were collected in parallel. Two biological replicates were collected for each genotype, and RNA isolation of five to eight adult males was performed as described above. Samples were normalized for RNA concentration and reverse-transcribed. The cDNA from each sample was used as template DNA in a PCR amplification of the Adh transcript. To distinguish the $A d h^{+}$and $A d h^{n 4}$ alleles, the PCR product was digested using PvuII and run on a $1 \%$ agarose gel (Supplemental Fig. S3). The gels were imaged using an Alpha Innotech Gel Doc System and quantitated using ImageJ (Abràmoff et al. 2004).

\section{Analysis of eya ${ }^{\text {cli-IID }}$}

eya $a^{\text {cli-IID }} \mathrm{cn}^{1} b w^{1} s p^{1} / C y O$ males were mated to e22c-GAL4, UAS$n l s D s R e d 2:: S V 40$ 3'UTR/CyO; FRT ${ }^{82 B \star}$, where * represents $S m g 6^{+}$, $S m g 6^{2 a}$, or $S m g 6^{292}$ virgins. Male progeny of genotype eya $a^{\text {cli-IID }} \mathrm{cn}^{1}$ $b w^{1} \quad s p^{1} /$ e22c-GAL4, UAS-nlsDsRed2::SV40 $3^{\prime} U T R ; \quad F R T^{82 B_{*} /+}$ were mated to virgins of genotype Btl-Gal4, UAS-GFP::SV40 $3^{\prime} U T R /+; D f(3 R) E D 6220 /+$. In the next generation, we collected larvae of genotype eya $a^{c l i-I I D} c n^{1} b w^{1} s p^{1} /$ Btl-Gal4, UAS-GFP::SV40 $3^{\prime} U T R ; F R T^{82 B} / D f(3 R) E D 6220$ based on increased GFP expression (due to the Smg6 mutation) and a lack of DsRed expression. A similar strategy was used on the X chromosome to collect larvae that were either $y w F R T^{19 A}$ or $y w U p f 2^{25 G} F R T^{19 A} / Y$; eya $a^{\text {cli-IID }} \mathrm{cn}^{1}$ $b w^{1} s p^{1} /$ e22c-GAL4, UAS-nlsDsRed2::SV40 3'UTR. RNA was isolated as described above, reverse-transcribed, and amplified using primers surrounding the eya $a^{\text {cli-IID }}$ PTC mutation. We determined the sequence of the RT-PCR product. eya ${ }^{\text {cli-IID }}$ stabilization was determined by measuring heights of the eya ${ }^{+}$and ey $a^{c l i-I I D}$ sequencing peaks.

\section{Microarray analysis}

RNA was isolated from male larvae collected from 0 to $4 \mathrm{~h}$ after the molt to third instar. To identify genotypes at this stage, we used balancers marked with fluorescent reporters: ActGFP for the $\mathrm{X}$ (Reichhart and Ferrandon 1998) and Dfd-EYFP for the third (Le et al. 2006). For Smg6 experiments, we crossed FM7i, $P\{$ ActGFP $\}$ JMR3/Y; Df(3R)ED6220/ TM6B, $P\{D f d-E Y F P\} \quad S b^{1}$ $c a^{1}$ males to either $w$; FRT $T^{82 B} / T M 6 B, P\{D f d-E Y F P\} S b^{1} c a^{1}$ or w; Smg6 $6^{2 a} / T M 6 B, P\{D f d-E Y F P\} S b^{1} c a^{1}$, or $w ; S m g 6^{292} / T M 6 B$, $P\{D f d-E Y F P\} S b^{1} c a^{1}$ virgins. For the X-chromosome NMD genes, we crossed FM7i, $P\{A c t G F P\} J M R 3 / Y$ males to either $y w$ $F R T^{19 A}$ or $y w S m g 1^{32 A P} F R T^{19 A}$, or $y w U p f 2^{25 G} F R T^{19 A} / F M 7 i$, $P\{A c t G F P\} J M R 3$ virgins. Progeny from these crosses were staged, and the appropriate genotypes were collected by scoring for the absence of the fluorescent balancers. RNA from two biological replicates of each genotype was reverse-transcribed, and the cDNA was labeled with either Cy3 or Cy5 and hybridized to Agilent 44K 2-color arrays. Data files were produced using Agilent's feature extraction software version 10.5. We normalized each array to total signal.

\section{Smg6 viability analysis}

To test the viability of $S m g 6$ alleles, virgin $F R T^{82 B}$ or $F R T^{82 B} S m g 6^{*}$ । TM6B, $P\{D f d-E Y F P\} S b^{1} c a^{l}$ flies were mated to $D f(3 R) E D 6220 /$ $T M 6 B, P\{D f d-E Y F P\} S b^{1} c a^{1}$ males, and progeny adults were analyzed for the presence and absence of the $S b$ marker associated with the balancer chromosome.

\section{Eye clones}

Mosaic eyes were generated by crossing $w$; ey-FLP; FRT $T^{82 B} P\{w+$ $r y+\} 90 E$ virgins to $F R T^{82 B}$ or $F R T^{82 B *}$ (where ${ }^{*}$ is EMS-generated mutation) males. Images at multiple planes were taken on an Axioskop2 plus (Zeiss) microscope and analyzed using an ImageJ stack analyzer and a stack focuser plug-in. Entirely mutant eyes were generated by the GMR-hid technique (Stowers and Schwarz 1999).

\section{SUPPLEMENTAL MATERIAL}

Supplemental material is available for this article.

\section{ACKNOWLEDGMENTS}

We thank Michael Galko for UAS-nls:DsRed transgenes; Alex Chapin and Jon Nelson for unpublished data; Shanhong Cheng, 
Sarah Elliott, and Deborah Johnson for assistance in screening; Barry Moore and Mark Yandell for help with array bioinformatics; the Metzstein and Thummel laboratories for useful discussions; and Gillian Stanfield, Carl Thummel, and members of the Metzstein laboratory for their comments on the manuscript. Fly stocks were obtained from the Bloomington Drosophila Stock Center. This work was supported by National Institutes of Health (NIH) University of Utah Developmental Biology Training Grant 5T32-HD07491 (to K.A.F.) and NIH 1R01GM084011 and a March of Dimes Award 5-FY07-664 (to M.M.M).

Received February 20, 2012; accepted June 7, 2012.

\section{REFERENCES}

Abràmoff MD, Magalhães PJ, Ram SJ. 2004. Image processing with ImageJ. Biophotonics international 11: 36-42.

Amrani N, Ganesan R, Kervestin S, Mangus DA, Ghosh S, Jacobson A. 2004. A faux 3'-UTR promotes aberrant termination and triggers nonsense-mediated mRNA decay. Nature 432: 112-118.

Anastasaki C, Longman D, Capper A, Patton EE, Cáceres JF. 2011. Dhx34 and Nbas function in the NMD pathway and are required for embryonic development in zebrafish. Nucleic Acids Res 39: 3686-3694.

Aronoff R, Baran R, Hodgkin J. 2001. Molecular identification of $s m g-4$, required for mRNA surveillance in C. elegans. Gene 268: 153-164.

Avery P, Vicente-Crespo M, Francis D, Nashchekina O, Alonso CR, Palacios IM. 2011. Drosophila Upf1 and Upf2 loss of function inhibits cell growth and causes animal death in a Upf3-independent manner. RNA 17: 624-638.

Brand AH, Perrimon N. 1993. Targeted gene expression as a means of altering cell fates and generating dominant phenotypes. Development 118: 401-415.

Brogna S. 1999. Nonsense mutations in the alcohol dehydrogenase gene of Drosophila melanogaster correlate with an abnormal $3^{\prime}$ end processing of the corresponding pre-mRNA. RNA 5: 562-573.

Bui QT, Zimmerman JE, Liu H, Bonini NM. 2000. Molecular analysis of Drosophila eyes absent mutants reveals features of the conserved Eya domain. Genetics 155: 709-720.

Cali BM, Anderson P. 1998. mRNA surveillance mitigates genetic dominance in Caenorhabditis elegans. Mol Gen Genet 260: 176-184.

Cali BM, Kuchma SL, Latham J, Anderson P. 1999. smg-7 is required for mRNA surveillance in Caenorhabditis elegans. Genetics 151: 605-616.

Chan W-K, Huang L, Gudikote JP, Chang Y-F, Imam JS, MacLean JA, Wilkinson MF. 2007. An alternative branch of the nonsensemediated decay pathway. EMBO J 26: 1820-1830.

Chang Y-F, Imam JS, Wilkinson MF. 2007. The nonsense-mediated decay RNA surveillance pathway. Annu Rev Biochem 76: 51-74.

Chen C-YA, Shyu A-B. 2003. Rapid deadenylation triggered by a nonsense codon precedes decay of the RNA body in a mammalian cytoplasmic nonsense-mediated decay pathway. Mol Cell Biol 23: $4805-4813$.

Chen Z, Smith KR, Batterham P, Robin C. 2005. Smg1 nonsense mutations do not abolish nonsense-mediated mRNA decay in Drosophila melanogaster. Genetics 171: 403-406.

Chia W, Savakis C, Karp R, Ashburner M. 1987. Adh ${ }^{n 4}$ of Drosophila melanogaster is a nonsense mutation. Nucleic Acids Res 15: 3931. doi: 10.1093/nar/15.9.3931.

Chintapalli VR, Wang J, Dow JAT. 2007. Using FlyAtlas to identify better Drosophila melanogaster models of human disease. Nat Genet 39: 715-720.

Chiu S-Y, Serin G, Ohara O, Maquat LE. 2003. Characterization of human Smg5/7a: A protein with similarities to Caenorhabditis elegans SMG5 and SMG7 that functions in the dephosphorylation of Upf1. RNA 9: 77-87.
Clerici M, Mourão A, Gutsche I, Gehring NH, Hentze MW, Kulozik A, Kadlec J, Sattler M, Cusack S. 2009. Unusual bipartite mode of interaction between the nonsense-mediated decay factors, UPF1 and UPF2. EMBO J 28: 2293-2306.

Cui Y, Hagan KW, Zhang S, Peltz SW. 1995. Identification and characterization of genes that are required for the accelerated degradation of mRNAs containing a premature translational termination codon. Genes Dev 9: 423-436.

Duffy JB, Harrison DA, Perrimon N. 1998. Identifying loci required for follicular patterning using directed mosaics. Development 125: 2263-2271.

Eberle AB, Stalder L, Mathys H, Zamudio Orozco R, Mühlemann O. 2008. Posttranscriptional gene regulation by spatial rearrangement of the 3' untranslated region. PLoS Biol 6: e92. doi: 10.1371/ journal.pbio.0060092.

Eberle AB, Lykke-Andersen S, Mühlemann O, Jensen TH. 2009. SMG6 promotes endonucleolytic cleavage of nonsense mRNA in human cells. Nat Struct Mol Biol 16: 49-55.

Fukuhara N, Ebert J, Unterholzner L, Lindner D, Izaurralde E, Conti E. 2005. SMG7 is a 14-3-3-like adaptor in the nonsense-mediated mRNA decay pathway. Mol Cell 17: 537-547.

Gatfield D, Izaurralde E. 2004. Nonsense-mediated messenger RNA decay is initiated by endonucleolytic cleavage in Drosophila. Nature 429: 575-578.

Gatfield D, Unterholzner L, Ciccarelli FD, Bork P, Izaurralde E. 2003. Nonsense-mediated mRNA decay in Drosophila: At the intersection of the yeast and mammalian pathways. EMBO J 22: 39603970.

Glavan F, Behm-Ansmant I, Izaurralde E, Conti E. 2006. Structures of the PIN domains of SMG6 and SMG5 reveal a nuclease within the mRNA surveillance complex. EMBO J 25: 5117-5125.

Grimson A, O'Connor S, Newman CL, Anderson P. 2004. SMG-1 is a phosphatidylinositol kinase-related protein kinase required for nonsense-mediated mRNA decay in Caenorhabditis elegans. Mol Cell Biol 24: 7483-7490.

He F, Jacobson A. 1995. Identification of a novel component of the nonsense-mediated mRNA decay pathway by use of an interacting protein screen. Genes Dev 9: 437-454.

He F, Brown AH, Jacobson A. 1997. Upf1p, Nmd2p, and Upf3p are interacting components of the yeast nonsense-mediated mRNA decay pathway. Mol Cell Biol 17: 1580-1594.

He F, Li X, Spatrick P, Casillo R, Dong S, Jacobson A. 2003. Genomewide analysis of mRNAs regulated by the nonsense-mediated and 5' to 3' mRNA decay pathways in yeast. Mol Cell 12: 1439-1452.

Hodgkin J, Papp A, Pulak R, Ambros V, Anderson P. 1989. A new kind of informational suppression in the nematode Caenorhabditis elegans. Genetics 123: 301-313.

Huang L, Lou C-H, Chan W, Shum EY, Shao A, Stone E, Karam R, Song H-W, Wilkinson MF. 2011. RNA homeostasis governed by cell type-specific and branched feedback loops acting on NMD. Mol Cell 43: 950-961.

Huntzinger E, Kashima I, Fauser M, Saulière J, Izaurralde E. 2008. SMG6 is the catalytic endonuclease that cleaves mRNAs containing nonsense codons in metazoan. RNA 14: 2609-2617.

Hwang J, Maquat LE. 2011. Nonsense-mediated mRNA decay (NMD) in animal embryogenesis: To die or not to die, that is the question. Curr Opin Genet Dev 21: 422-430.

Kadlec J, Izaurralde E, Cusack S. 2004. The structural basis for the interaction between nonsense-mediated mRNA decay factors UPF2 and UPF3. Nat Struct Mol Biol 11: 330-337.

Kashima I, Yamashita A, Izumi N, Kataoka N, Morishita R, Hoshino S, Ohno M, Dreyfuss G, Ohno S. 2006. Binding of a novel SMG-1Upf1-eRF1-eRF3 complex (SURF) to the exon junction complex triggers Upf1 phosphorylation and nonsense-mediated mRNA decay. Genes Dev 20: 355-367.

Kashima I, Jonas S, Jayachandran U, Buchwald G, Conti E, Lupas AN, Izaurralde E. 2010. SMG6 interacts with the exon junction complex via two conserved EJC-binding motifs (EBMs) required for nonsense-mediated mRNA decay. Genes Dev 24: 2440-2450. 
Kerényi Z, Mérai Z, Hiripi L, Benkovics A, Gyula P, Lacomme C, Barta E, Nagy F, Silhavy D. 2008. Inter-kingdom conservation of mechanism of nonsense-mediated mRNA decay. EMBO J 27: 1585-1595.

Kertész S, Kerényi Z, Mérai Z, Bartos I, Pálfy T, Barta E, Silhavy D. 2006. Both introns and long $3^{\prime}$-UTRs operate as cis-acting elements to trigger nonsense-mediated decay in plants. Nucleic Acids Res 34: 6147-6157.

Le T, Liang Z, Patel H, Yu MH, Sivasubramaniam G, Slovitt M, Tanentzapf G, Mohanty N, Paul SM, Wu VM, et al. 2006. A new family of Drosophila balancer chromosomes with a $w^{-} d f d$-GMR yellow fluorescent protein marker. Genetics 174: 2255-2257.

Lee BS, Culbertson MR. 1995. Identification of an additional gene required for eukaryotic nonsense mRNA turnover. Proc Natl Acad Sci 92: 10354-10358.

Leeds P, Peltz SW, Jacobson A, Culbertson MR. 1991. The product of the yeast UPF1 gene is required for rapid turnover of mRNAs containing a premature translational termination codon. Genes Dev 5: 2303-2314.

Leeds P, Wood JM, Lee BS, Culbertson MR. 1992. Gene products that promote mRNA turnover in Saccharomyces cerevisiae. Mol Cell Biol 12: $2165-2177$.

Lejeune F, Maquat LE. 2005. Mechanistic links between nonsensemediated mRNA decay and pre-mRNA splicing in mammalian cells. Curr Opin Cell Biol 17: 309-315.

Lejeune F, Li X, Maquat LE. 2003. Nonsense-mediated mRNA decay in mammalian cells involves decapping, deadenylating, and exonucleolytic activities. Mol Cell 12: 675-687.

Longman D, Plasterk RHA, Johnstone IL, Cáceres JF. 2007. Mechanistic insights and identification of two novel factors in the $C$. elegans NMD pathway. Genes Dev 21: 1075-1085.

Luke B, Azzalin CM, Hug N, Deplazes A, Peter M, Lingner J. 2007. Saccharomyces cerevisiae Ebslp is a putative ortholog of human Smg7 and promotes nonsense-mediated mRNA decay. Nucleic Acids Res 35: 7688-7697.

Metzstein MM, Krasnow MA. 2006. Functions of the nonsensemediated mRNA decay pathway in Drosophila development. PLoS Genet 2: e180. doi: 10.1371/journal.pgen.0020180.

Muhlrad D, Parker R. 1994. Premature translational termination triggers mRNA decapping. Nature 370: 578-581.

Newsome TP, Asling B, Dickson BJ. 2000. Analysis of Drosophila photoreceptor axon guidance in eye-specific mosaics. Development 127: $851-860$.
Okada-Katsuhata Y, Yamashita A, Kutsuzawa K, Izumi N, Hirahara F, Ohno S. 2012. N- and C-terminal Upf1 phosphorylations create binding platforms for SMG-6 and SMG-5:SMG-7 during NMD. Nucleic Acids Res 40: 1251-1266.

Page MF, Carr B, Anders KR, Grimson A, Anderson P. 1999. SMG-2 is a phosphorylated protein required for mRNA surveillance in Caenorhabditis elegans and related to Upflp of yeast. Mol Cell Biol 19: 5943-5951.

Rehwinkel J, Letunic I, Raes J, Bork P, Izaurralde E. 2005. Nonsensemediated mRNA decay factors act in concert to regulate common mRNA targets. RNA 11: 1530-1544.

Reichhart JM, Ferrandon D. 1998. Green balancers. Drosoph Inf Serv 81: 201-202.

Ryder E, Ashburner M, Bautista-Llacer R, Drummond J, Webster J, Johnson G, Morley T, Chan YS, Blows F, Coulson D, et al. 2007. The DrosDel deletion collection: A Drosophila genomewide chromosomal deficiency resource. Genetics 177: 615-629.

Serin G, Gersappe A, Black JD, Aronoff R, Maquat LE. 2001. Identification and characterization of human orthologues to Saccharomyces cerevisiae Upf2 protein and Upf3 protein (Caenorhabditis elegans SMG-4). Mol Cell Biol 21: 209-223.

Shiga Y, Tanaka-Matakatsu M, Hayashi S. 1996. A nuclear GFP/ $\beta$-galactosidase fusion protein as a marker for morphogenesis in living Drosophila. Dev Growth Differ 38: 99-106.

Stowers RS, Schwarz TL. 1999. A genetic method for generating Drosophila eyes composed exclusively of mitotic clones of a single genotype. Genetics 152: 1631-1639.

Swisher KD, Parker R. 2011. Interactions between Upf1 and the decapping factors Edc3 and Pat1 in Saccharomyces cerevisiae. PLoS ONE 6: e26547. doi: 10.1371/journal.pone.0026547.

Takeshita D, Zenno S, Lee WC, Saigo K, Tanokura M. 2007. Crystal structure of the PIN domain of human telomerase-associated protein EST1A. Proteins 68: 980-989.

Wittkopp N, Huntzinger E, Weiler C, Sauliere J, Schmidt S, Sonawane M, Izaurralde E. 2009. Nonsense-mediated mRNA decay effectors are essential for zebrafish embryonic development and survival. Mol Cell Biol 29: 3517-3528.

Xu T, Rubin GM. 1993. Analysis of genetic mosaics in developing and adult Drosophila tissues. Development 117: 1223-1237.

Yepiskoposyan H, Aeschimann F, Nilsson D, Okoniewski M, Mühlemann O. 2011. Autoregulation of the nonsense-mediated mRNA decay pathway in human cells. RNA 17: 2108-2118. 

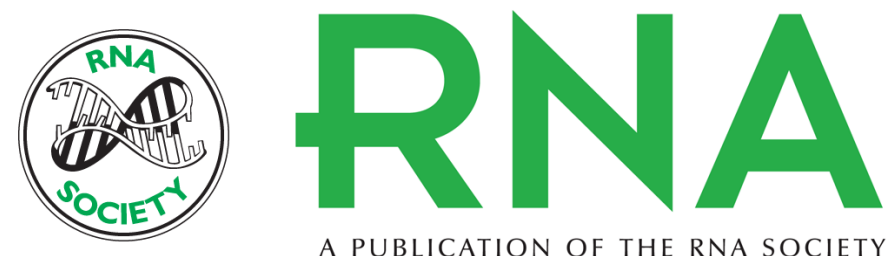

A PUBLICATION OF THE RNA SOCIETY

\section{Drosophila mutants show NMD pathway activity is reduced, but not eliminated, in the absence of Smg6}

Kimberly A. Frizzell, Shawn G. Rynearson and Mark M. Metzstein

RNA 2012 18: 1475-1486 originally published online June 27, 2012

Access the most recent version at doi:10.1261/rna.032821.112

Supplemental
Material http://rnajournal.cshlp.org/content/suppl/2012/06/18/rna.032821.112.DC1

References This article cites 63 articles, 25 of which can be accessed free at: http://rnajournal.cshlp.org/content/18/8/1475.full.html\#ref-list-1

License

Email Alerting Receive free email alerts when new articles cite this article - sign up in the box at the Service top right corner of the article or click here. 